\title{
A simple case of rationality of escalation
}

\author{
Pierre Lescanne \\ University of Lyon, École normale supérieure de Lyon, CNRS (LIP), \\ 46 allée d'Italie, 69364 Lyon, France
}

\begin{abstract}
Escalation is the fact that in a game (for instance an auction), the agents play forever. It is not necessary to consider complex examples to establish its rationality. In particular, the 0,1-game is an extremely simple infinite game in which escalation arises naturally and rationally. In some sense, it can be considered as the paradigm of escalation. Through an example of economic games, we show the benefit economics can take of coinduction.
\end{abstract}

Keywords: economic game, infinite game, sequential game, crash, escalation, speculative bubble, coinduction, auction.

[T]he future of economics is increasingly technical work that is founded on the vision that the economy is a complex system.

David Collander [6]

Sequential games are the natural framework for decision processes. In this paper we study a decision phenomenon called escalation. Finite sequential games (also known as extensive games) have been introduced by Kuhn [9] and subgame perfect equilibria have been introduced by Selten [19] whereas escalation has been introduced by Shubik [20]. Sequential games are games in which each player plays one after the other (or possibly after herself). In some specific infinite games, it has been showed that escalation cannot occur among rational players. Here we show on a simple example, the 0,1 game, that this is not the case if one uses coinduction. In addition the 0,1 game has nice properties which make it an excellent paradigm of escalation and a good domain of application for coalgebras and coinduction.

\section{The problem of escalation}

That "rational agents" should not engage in such [es-
calation] behavior seems obvious.

Wolfgang Leininger [1]

Escalation in sequential games is a classic of game theory and it is admitted that escalation is irrational. The rationality which we consider is that given 
by equilibria. It has been proved that in finite sequential games, rationality is obtained by a specific equilibrium called backward induction (see Appendix). More precisely a consequence of Aumann's theorem [2] says that an agent takes a rational decision in a finite sequential game if she makes her choice according to backward induction. In this paper we generalize backward induction into subgame perfect equilibria and we consider naturally that rationality is reached by subgame perfect equilibria (SPE in short) relying on Capretta's [5] extension of Aumann's theorem.

What is escalation? In a sequential game, escalation is the possibility that agents take rational decisions forever without stopping. This phenomenon has been evidenced by Shubik [20] in a game called the dollar auction. Without being very difficult, its analysis is relatively involved, because it requires infinitely many strategy profiles indexed by $n \in \mathbb{N}[12$. Moreover in each step there are two and only two equilibria. By an observation of the past decisions of her opponent an agent could get a clue of her strategy and might this way avoid escalation. This blindness of the agents is perhaps not completely realistic and was criticized (see [14] Section 4.2). In this paper, we propose an example which is much simpler theoretically and which offers infinitely many equilibria at each step. Due to the form of the equilibria, the agent has no clue on which strategy is taken by her opponent.

Escalation and infinite games. Books and articles [7/8|17|11|16] which cover escalation take for granted that escalation is irrational. Following Shubik, all accept that escalation takes place and can only take place in an infinite game, but their argument uses a reasoning on finite games. Indeed, if one cuts the infinite game in which escalation is supposed to take place at a finite position, one gets a finite game, in which the only right and rational decision is to never start the game, because the only backward induction equilibrium corresponds to not start playing. Then the result is extrapolated by the authors to infinite games by making the size of the game to grow to infinity. However, it has been known for a long time at least since Weierstraß 24, that the "cut and extrapolate" method is wrong (see Appendix). For Weierstraß this would lead to the conclusion that the infinite sum of differentiable functions would be differentiable whereas he has exhibited a famous counterexample. In the case of infinite structures like infinite games, the right reasoning is coinduction. With coinduction we were able to show that the dollar auction has a rational escalation [15]14]. Currently, since the tools used generally in economics are pre-coinduction based, they conclude that bubbles and crises are impossible and everybody's experience has witnessed the opposite. Careful analysis done by quantitative economists, like for instance Bouchaud [34, have shown that bursts, which share much similarities with escalation, actually take place at any time scale. Escalation is therefore an intrinsic feature of economics. Consequently, coinduction is the tool that economists who call for a refoundation of economics [6]3] are waiting for [25]. 
Structure of the paper This paper is structured as follows. In Section 2 we present infinite games, infinite strategy profiles and infinite strategies, then we describe the 0,1-game in Section 3, Last, we introduce the concept of equilibrium (Sections 4 and 5) and we discuss escalation (Section 6). In an appendix, we talk about finite games and finite strategy profiles.

\section{Two choice sequential games}

Our aim is not to present a general theory. For this the reader is invited to look at 11415. But we want to give a taste of infinite sequential games through a very simple one. This game has two agents and two choices. To support our claim about the rationality of escalation, we do not need more features. In [14, we have shown the existence of a big conceptual gap between finite games and infinite games.

Assume that the set $\mathrm{P}$ of agents is made of two agents called $\mathrm{A}$ and $\mathrm{B}$. In this framework, an infinite sequential two choice game has two shapes. In the first shape, it is an ending position in which case it boils down to the distribution of the payoffs to the agents. In other words the game is reduced to a function $f: A \mapsto f_{\mathrm{A}}, B \mapsto f_{\mathrm{B}}$ and we write it $\langle f\rangle$. In the second shape, it is a generic game with a set $C$ made of two potential choices: $d$ or $r$ ( $d$ for down and $r$ for right). Since the game is potentially infinite, it may continue forever. Thus formally in this most general configuration a game can be seen as a triple:

$$
g=\left\langle p, g_{d}, g_{r}\right\rangle .
$$

where $p$ is an agent and $g_{d}$ and $g_{r}$ are themselves games. The subgame $g_{d}$ corresponds to the down choice, i.e., the choice corresponding to go down and the subgame $g_{r}$ corresponds to the right choice, i.e., the choices corresponding to go to the right. In other words, we define a functor:

$$
\langle\rangle: \mathrm{X} \rightarrow \text { Payoff }+\mathrm{P} \times \mathrm{X} \times \mathrm{X} .
$$

of which Game is the final coalgebra and where $P=\{A, B\}$ and Payoff $=\mathbb{R}^{P}$.

\section{$2.1 \quad$ Strategy profiles}

From a game, one can deduce strategy profiles (later we will also say simply profiles), which is obtained by adding a label, at each node, which is a choice made by the agent. A choice belong to the set $\{d, r\}$. In other words, a strategy profile is obtained from a game by adding, at each node, a new label, namely a choice. Therefore a strategy profile which does not correspond to an ending game is a quadruple:

$$
s=\left\langle\left\langle p, c, s_{d}, s_{r}\right\rangle\right\rangle,
$$

where $p$ is an agent (A or B), $c$ is choice ( $d$ or $r$ ), and, $s_{d}$ and $s_{r}$ are two strategy profiles. The strategy profile which corresponds to an ending position has no 
choice, namely it is reduced to a function $\langle\langle f\rangle\rangle=\left\langle\left\langle A \mapsto f_{\mathrm{A}}, B \mapsto f_{\mathrm{B}}\right\rangle\right\rangle$. From a strategy profile, one can build a game by removing the choices:

$$
\begin{aligned}
\operatorname{game}(\langle\langle f\rangle\rangle) & =\langle f\rangle \\
\operatorname{game}\left(\left\langle\left\langle p, c, s_{d}, s_{r}\right\rangle\right)\right) & =\left\langle p, \operatorname{game}\left(s_{d}\right), \operatorname{game}\left(s_{r}\right)\right\rangle
\end{aligned}
$$

game $(s)$ is the underlying game of the strategy profile $s$.

Given a strategy profile $s$, one can associate, by induction, a (partial) payoff function $\widehat{s}$, as follows:

$$
\begin{array}{ll}
\text { when } s=\langle\langle f\rangle\rangle & \widehat{s}=f \\
\text { when } s=\left\langle\left\langle p, d, s_{d}, s_{r}\right\rangle\right\rangle & \widehat{s}=\widehat{s_{d}} \\
\text { when } s=\left\langle\left\langle p, r, s_{d}, s_{r}\right\rangle\right\rangle & \widehat{s}=\widehat{s_{r}}
\end{array}
$$

$\widehat{s}$ is not defined if its definition runs in an infinite process. For instance, if $s_{\mathrm{A}, \infty}$ is the strategy profile defined in Section $6, \widehat{s_{\mathrm{A}, \infty}}$ is not defined. To ensure that we consider only strategy profiles where the payoff function is defined we restrict to strategy profiles that are called convergent, written $s \downarrow$ (or sometimes prefixed $\downarrow(s))$ and defined as the least predicate satisfying

$$
s=\langle\langle f\rangle\rangle \quad \vee \quad s=\left\langle\left\langle p, d, s_{d}, s_{r}\right\rangle\right\rangle \Rightarrow s_{d} \downarrow \wedge\left\langle\left\langle p, r, s_{d}, s_{r}\right\rangle\right\rangle \Rightarrow s_{r} \downarrow .
$$

Proposition 1. If $s \downarrow$, then $\widehat{s}$ is defined.

Proof. By induction. If $s=\langle\langle f\rangle\rangle$, then since $\widehat{s}=f$ and $f$ is defined, $\widehat{s}$ is defined.

If $s=\left\langle\left\langle p, c, s_{d}, s_{r}\right\rangle\right\rangle$, there are two cases: $c=d$ or $c=r$. Let us look at $c=d$. If $c=d, \widehat{s_{d}}$ is defined by induction and since $\widehat{s}=\widehat{s_{d}}$, we conclude that $\widehat{s}$ is defined.

The case $c=r$ is similar.

As we will consider the payoff function also for subprofiles, we want the payoff function to be defined on subprofiles as well. Therefore we define a property stronger than convergence which we call strong convergence. We say that a strategy profile $s$ is strongly convergent and we write it $s \Downarrow$ if it is the largest predicate fulfilling the following conditions.

$-\left\langle\left\langle p, c, s_{d}, s_{r}\right\rangle\right\rangle \Downarrow$ if

- $\left\langle\left\langle p, c, s_{d}, s_{r}\right\rangle\right\rangle$ is convergent,

- $s_{d}$ is strongly convergent,

- $s_{r}$ is strongly convergent.

- $\langle\langle f\rangle\rangle$ is always strongly convergent

More formally:

$$
s \Downarrow \quad \Leftrightarrow \quad \Leftrightarrow \quad s=\langle\langle f\rangle\rangle \vee\left(s=\left\langle\left\langle p, c, s_{d}, s_{r}\right\rangle\right\rangle \wedge s \downarrow \wedge s_{d} \Downarrow \wedge s_{r} \Downarrow\right) .
$$


There is however a difference between the definitions of $\downarrow$ and $\Downarrow$. Wherever $s \downarrow$ is defined by induction 1 , from the ending games to the game, $s \Downarrow$ is defined by coinduction 2 .

Both concepts are based on the fixed-point theorem established by Tarski [21]. The definition of $\Downarrow$ is typical of infinite games and means that $\Downarrow$ is invariant along the infinite game. To make the difference clear between the definitions, we use the symbol $\Leftrightarrow \subset \rightarrow$ for inductive definitions and the symbol $\Leftrightarrow \subset \Rightarrow$ for coinductive definitions. By the way, the definition of the function game is also coinductive.

We can define the notion of subprofile, written $\precsim$ :

$$
s^{\prime} \precsim s \quad \Leftrightarrow \subset \Rightarrow \quad s^{\prime} \sim_{s} s \vee s=\left\langle\left\langle p, c, s_{d}, s_{r}\right\rangle\right\rangle \wedge\left(s^{\prime} \precsim s_{d} \vee s^{\prime} \precsim s_{r}\right),
$$

where $\sim_{s}$ is the bisimilarity among profiles defined as the largest binary predicate $s^{\prime} \sim_{s} s$ such that

$s^{\prime}=\langle\langle f\rangle\rangle=s \quad \vee \quad\left(s^{\prime}=\left\langle\left\langle p, c, s_{d}^{\prime}, s_{r}^{\prime}\right\rangle\right\rangle \wedge s=\left\langle\left\langle p, c, s_{d}, s_{r}\right\rangle\right\rangle \wedge s_{d}^{\prime} \sim_{s} s_{d} \wedge s_{r}^{\prime} \sim_{s} s_{r}\right)$.

Notice that since we work with infinite objects, we may have $s \chi_{s} s^{\prime}$ and $s \precsim s^{\prime} \precsim s$. In other words, an infinite profile can be a strict subprofile of itself. This is the case for $s_{1,0, a}$ and $s_{1,0, b}$ in Section 4. If a profile is strongly convergent, then the payoffs associated with all its subprofiles are defined.

Proposition 2. If $s_{1} \Downarrow$ and if $s_{2} \precsim s_{1}$, then $\widehat{s_{2}}$ is defined.

\subsection{The always modality}

We notice that $\downarrow$ characterizes a profile by a property of the head node, we would say that this property is local. $\Downarrow$ is obtained by distributing the property along the game. In other words we transform the predicate $\downarrow$ and such a predicate transformer is called a modality. Here we are interested by the modality always, also written

Given a predicate $\Phi$ on strategy profiles, the predicate $\square P$ is defined coinductively as follows:

$$
\square \Phi(s) \Leftrightarrow \bigodot \Rightarrow \Phi(s) \wedge s=\left\langle\left\langle p, c, s_{d}, s_{r}\right\rangle\right\rangle \Rightarrow\left(\square \Phi\left(s_{d}\right) \wedge \square \Phi\left(s_{r}\right)\right) .
$$

The predicate "is strongly convergent" is the same as the predicate "is always convergent".

Proposition 3. $s \Downarrow \quad \Leftrightarrow \quad \square \downarrow(s)$.

\footnotetext{
${ }^{1}$ Roughly speaking a definition by induction works from the basic elements, here the ending games, to constructed elements.

${ }^{2}$ Roughly speaking a definition by coinduction works on infinite objects, like an invariant.
} 


\section{$2.3 \quad$ Strategies}

The coalgebra of strategies 3 is defined by the functor

$$
\llbracket \rrbracket: X \rightarrow \mathbb{R}^{\mathrm{P}}+(\mathrm{P}+\text { Choice }) \times X \times X
$$

where Choice $=\{d, r\}$. A strategy of agent $p$ is a game in which some occurrences of $p$ are replaced by choices. A strategy is written $\llbracket f \rrbracket$ or $\llbracket x, s_{1}, s_{2} \rrbracket$. By replacing the choice made by agent $p$ by the agent $p$ herself, we can associate a game with a pair consisting of a strategy and an agent:

$$
\begin{aligned}
& \operatorname{st} 2 \mathrm{~g}(\llbracket f \rrbracket, p) \quad=\langle f\rangle \\
& \operatorname{st} 2 \mathrm{~g}\left(\llbracket x, s t_{1}, s t_{2} \rrbracket, p\right)=\text { if } x \in \mathrm{P} \text { then }\left\langle x, \operatorname{st} 2 \mathrm{~g}\left(s t_{1}, p\right), \operatorname{st} 2 \mathrm{~g}\left(s t_{2}, p\right)\right\rangle \\
& \text { else }\left\langle p, \operatorname{st} 2 \mathrm{~g}\left(s t_{1}, p\right), \operatorname{st} 2 \mathrm{~g}\left(s t_{2}, p\right)\right\rangle \text {. }
\end{aligned}
$$

If a strategy st is really the strategy of agent $p$ it should contain nowhere $p$ and should contain a choice $c$ instead. In this case we say that st is full for $p$ and we write it $s t \Psi^{p}$.

$$
\begin{aligned}
& \llbracket f \rrbracket \rrbracket^{p}
\end{aligned}
$$

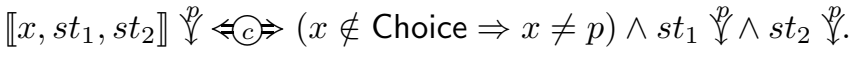

We can sum strategies to make a strategy profile. But for that we have to assume that all strategies are full and underlie the same game. In other words, $\left(s t_{p}\right)_{p \in \mathrm{P}}$ is a family of strategies such that:

$-\forall p \in \mathrm{P}, s t_{p}{ }^{p}$

- there exists a game $g$ such that $\forall p \in \mathrm{P}, \operatorname{st} 2 \mathrm{~g}\left(s t_{p}\right)=g$.

We define $\bigoplus_{p \in \mathrm{P}} s t_{p}$ as follows:

$$
\begin{aligned}
& \bigoplus_{p \in \mathrm{P}} \llbracket f \rrbracket=\langle\langle f\rangle\rangle \\
& \llbracket c, s t_{p^{\prime}, 1}, s t_{p^{\prime}, 2} \rrbracket \oplus \bigoplus_{p \in \mathrm{P} \backslash p^{\prime}} \llbracket p^{\prime}, s t_{p, 1}, s t_{p, 2} \rrbracket=\left\langle\left\langle p^{\prime}, c, \bigoplus_{p \in \mathrm{P}} s t_{p, 1}, \bigoplus_{p \in \mathrm{P}} s t_{p, 2}\right\rangle\right\rangle .
\end{aligned}
$$

We can show that the game underlying all the strategies is the game underlying the strategy profile which is the sum of the strategies.

Proposition 4. st2g $\left(s t_{p^{\prime}}, p^{\prime}\right)=\operatorname{game}\left(\bigoplus_{p \in P} s t_{p}\right)$.

\footnotetext{
${ }^{3}$ A strategy is not the same as a strategy profile, which is obtained as the sum of strategies.
} 


\section{Comb games and the 0,1-game}

We will restrict to simple games which have the shape of combs,

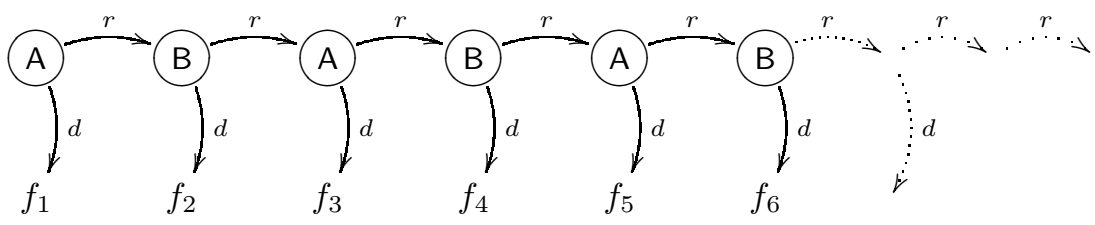

At each step the agents have only two choices, namely to stop or to continue. Let us call such a game, a comb game.

We introduce infinite games by means of equations. Let us see how this applies to define the 0,1-game. First consider two payoff functions:

$$
\begin{aligned}
& f_{0,1}=\mathrm{A} \mapsto 0, \mathrm{~B} \mapsto 1 \\
& f_{1,0}=\mathrm{A} \mapsto 1, \mathrm{~B} \mapsto 0
\end{aligned}
$$

we define two games

$$
\begin{aligned}
& G_{0,1}=\left\langle\mathrm{A},\left\langle f_{0,1}\right\rangle, G_{1,0}\right\rangle \\
& G_{1,0}=\left\langle\mathrm{B},\left\langle f_{1,0}\right\rangle, G_{0,1}\right\rangle
\end{aligned}
$$

This means that we define an infinite sequential game $G_{0,1}$ in which agent A is the first player and which has two subgames: the trivial game $\left\langle f_{1,0}\right\rangle$ and the game $G_{1,0}$ defined in the other equation. The game $G_{0,1}$ can be pictured as follows:

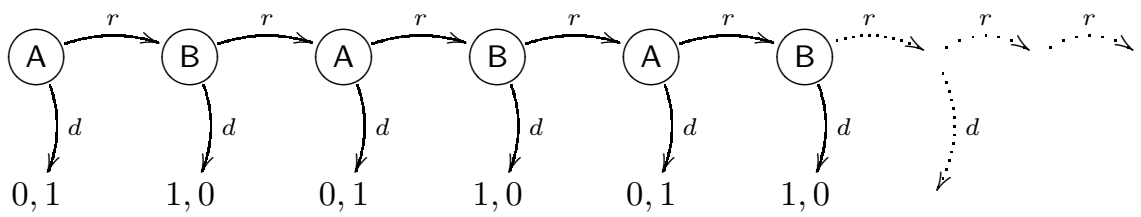

or more simply in Figure 1. a.

From now on, we assume that we consider only strategy profiles whose underlying game is the 0,1-game. They are characterized by the following predicates

$$
\begin{aligned}
& \mathrm{S}_{0}(s) \Leftrightarrow \Subset \Leftrightarrow s=\left\langle\left\langle\mathrm{A}, c, f_{0,1}, s^{\prime}\right\rangle\right\rangle \wedge \mathrm{S}_{1}\left(s^{\prime}\right) \\
& \mathrm{S}_{1}(s) \Leftrightarrow \Subset \Rightarrow s=\left\langle\left\langle\mathrm{B}, c, f_{1,0}, s^{\prime}\right\rangle\right\rangle \wedge \mathrm{S}_{0}\left(s^{\prime}\right) .
\end{aligned}
$$

Notice that the 0,1-game we consider is somewhat a zero-sum game, but we are not interested in this aspect. Moreover, a very specific instance of a 0,1 game has been considered (by Ummels [22] for instance), but these authors are not interested in the general structure of the game, but in a specific model on a finite graph, which is not general enough for our taste. Therefore this is not a direct generalization of finite sequential games (replacing induction by coinduction) and this not a framework to study escalation. 


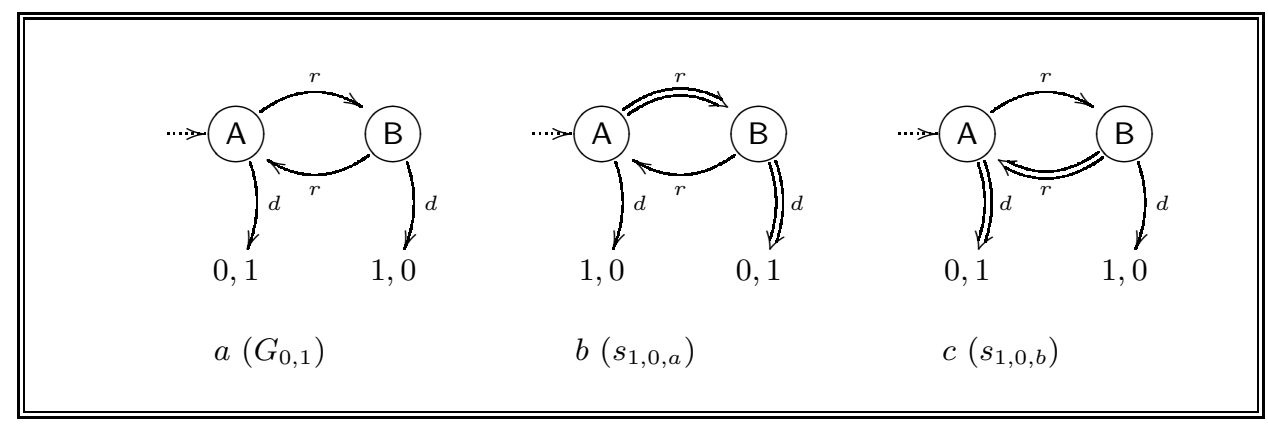

Fig. 1. The 0,1-game and two equilibria seen compactly

\section{Subgame perfect equilibria}

Among the strategy profiles, one can select specific ones that are called subgame perfect equilibria. Subgame perfect equilibria are specific strategy profiles that fulfill a predicate SPE. This predicate relies on another predicate PE which checks a local property.

$$
\begin{aligned}
\mathrm{PE}(s) \Leftrightarrow \quad \Leftrightarrow \Downarrow & \wedge s=\left\langle\left\langle p, d, s_{d}, s_{r}\right\rangle\right\rangle \Rightarrow \widehat{s_{d}}(p) \geq \widehat{\widehat{s_{r}}}(p) \\
\wedge s & =\left\langle\left\langle p, r, s_{d}, s_{r}\right\rangle\right\rangle \Rightarrow \widehat{s_{r}}(p) \geq \widehat{s_{d}}(p)
\end{aligned}
$$

A strategy profile is a subgame perfect equilibrium if the property PE holds always:

$$
\mathrm{SPE}=\square \mathrm{PE} .
$$

We may now wonder what the subgame perfect equilibria of the 0,1-game are. We present two of them in Figure 1 b and 1 c. But there are others. To present them, let us define a predicate "A continues and B eventually stops"

$$
\begin{aligned}
\operatorname{AcBes}(s) \Leftrightarrow(i) \Rightarrow s=\left\langle\left\langle p, c,\langle\langle f\rangle\rangle, s^{\prime}\right\rangle\right\rangle \Rightarrow & \left(p=\mathrm{A} \wedge f=f_{0,1} \wedge c=r \wedge \operatorname{AcBes}\left(s^{\prime}\right)\right) \vee \\
& \left(p=\mathrm{B} \wedge f=f_{1,0} \wedge\left(c=d \vee \operatorname{AcBes}\left(s^{\prime}\right)\right)\right.
\end{aligned}
$$

Proposition 5. $\left(\mathrm{S}_{1}(s) \vee \mathrm{S}_{0}(s)\right) \Rightarrow \operatorname{AcBes}(s) \Rightarrow \widehat{s}=f_{1,0}$

Proof. If $s=\left\langle\left\langle p, c,\langle\langle f\rangle\rangle, s^{\prime}\right\rangle\right\rangle$, then $\mathrm{S}_{0}\left(s^{\prime}\right) \vee \mathrm{S}_{1}\left(s^{\prime}\right)$. Therefore if $\operatorname{AcBes}\left(s^{\prime}\right)$, by induction, $\widehat{s^{\prime}}=f_{0,1}$. By case:

- If $p=\mathrm{A} \wedge c=r$, then $\operatorname{AcBes}\left(s^{\prime}\right)$ and by definition of $\widehat{s}$, we have $\widehat{s}=\widehat{s^{\prime}}=f_{0,1}$

- if $p=\mathrm{B} \wedge c=d$, the $\widehat{s}=\widehat{\left\langle\left\langle f_{0,1}\right\rangle\right\rangle}=f_{0,1}$.

- if $p=\mathrm{B} \wedge c=r$, then $\operatorname{AcBes}\left(s^{\prime}\right)$ and by definition of $\widehat{s}, \widehat{s}=\widehat{s^{\prime}}=f_{0,1}$.

Like we generalize PE to SPE by applying the modality $\square$, we generalize AcBes into SAcBes by stating:

$$
\text { SAcBes }=\square \text { AcBes. }
$$


There are at least two profiles which satisfies SAcBes namely $s_{1,0, a}$ and $s_{1,0, b}$ which have been studied in [14 and pictured in Figure 1.

$$
\begin{array}{ll}
s_{1,0, a} \Leftrightarrow C \Rightarrow\left\langle\left\langle\mathrm{A}, r, f_{0,1}, s_{1,0, b}\right\rangle\right\rangle & \left.s_{1,0, b} \Leftrightarrow C \Rightarrow\left\langle\mathrm{B}, d, f_{1,0}, s_{1,0, a}\right\rangle\right\rangle \\
s_{0,1, a} \Leftrightarrow C \Rightarrow\left\langle\left\langle\mathrm{A}, d, f_{0,1}, s_{0,1, b}\right\rangle\right\rangle & s_{0,1, b} \Leftrightarrow C \Rightarrow\left\langle\left\langle\mathrm{B}, r, f_{1,0}, s_{0,1, a}\right\rangle\right\rangle
\end{array}
$$

Proposition 6. $\operatorname{SAcBes}(s) \Rightarrow s \Downarrow$.

We may state the following proposition.

Proposition 7. $\forall s,\left(\mathrm{~S}_{0}(s) \vee \mathrm{S}_{1}(s)\right) \Rightarrow(\operatorname{SAcBes}(s) \Rightarrow \mathrm{SPE}(s))$.

Proof. Since SPE is a coinductively defined predicate, the proof is by coinduction.

Given an $s$, we have to prove $\forall s, \square \operatorname{AcBes}(s) \wedge\left(\mathrm{S}_{0}(s) \vee \mathrm{S}_{1}(s)\right) \Rightarrow \square \mathrm{PE}(s)$.

For that we assume $\square \operatorname{AcBes}(s) \wedge\left(\mathrm{S}_{0}(s) \vee \mathrm{S}_{1}(s)\right)$ and in addition (coinduction principle) $\square \mathrm{PE}\left(s^{\prime}\right)$ for all strict subprofiles $s^{\prime}$ of $s$ and we prove $\operatorname{PE}(s)$. In other words, $s \Downarrow \wedge\left\langle\left\langle p, d, s_{d}, s_{r}\right\rangle\right\rangle \Rightarrow \widehat{s_{d}}(p) \geq \widehat{s_{r}}(p) \wedge\left\langle\left\langle p, r, s_{d}, s_{r}\right\rangle\right\rangle \Rightarrow \widehat{s_{r}}(p) \geq \widehat{s_{d}}(p)$.

By Proposition 6 we have $s \Downarrow$.

By Proposition 5, we know that for every subprofile $s^{\prime}$ of a profile $s$ that satisfies $\mathrm{S}_{1}(s) \vee \mathrm{S}_{0}(s)$ we have $\widehat{s^{\prime}}=f_{1,0}$ except when $s^{\prime}=\left\langle\left\langle f_{0,1}\right\rangle\right\rangle$. Let us prove $\left\langle\left\langle p, d, s_{d}, s_{r}\right\rangle\right\rangle \Rightarrow \widehat{s_{d}}(p) \geq \widehat{s_{r}}(p) \wedge\left\langle\left\langle p, r, s_{d}, s_{r}\right\rangle\right\rangle \Rightarrow \widehat{s_{r}}(p) \geq \widehat{s_{d}}(p)$. Let us proceed by case:

$-s=\left\langle\left\langle\mathrm{A}, r,\left\langle\left\langle f_{0,1}\right\rangle\right\rangle, s^{\prime}\right\rangle\right\rangle$. Then $\mathrm{S}_{0}(s)$ and $\mathrm{S}_{1}\left(s^{\prime}\right)$. Since $\square \operatorname{AcBes}(s)$, we have $\operatorname{AcBes}\left(s^{\prime}\right)$, therefore $\widehat{s^{\prime}}=f_{1,0}$ hence $\widehat{s^{\prime}}(\mathrm{A})=1$ and $f_{0,1}(\mathrm{~A})=0$, henceforth $\widehat{s^{\prime}}(\mathrm{A}) \geq f_{0,1}(\mathrm{~A})$.

$-s=\left\langle\left\langle\mathrm{B}, r,\left\langle\left\langle f_{1,0}\right\rangle\right\rangle, s^{\prime}\right\rangle\right\rangle$. Then $\mathrm{S}_{1}(s)$ and $\mathrm{S}_{0}\left(s^{\prime}\right)$. Since $\square \operatorname{AcBes}(s)$, we have $\operatorname{AcBes}\left(s^{\prime}\right)$, therefore $\widehat{s^{\prime}}=f_{1,0}$ hence $\widehat{s^{\prime}}(\mathrm{B})=0$ and $f_{1,0}(\mathrm{~B})=0$, henceforth $\widehat{s^{\prime}}(\mathrm{B}) \geq f_{1,0}(\mathrm{~B})$.

Symmetrically we can define a predicate BcAes for "B continues and A eventually stops" and a predicate SBcAes which is SBcAes $=\square$ BcAes which means that B continues always and A stops infinitely often. With the same argument as for SAcBes one can conclude that

$$
\forall s,\left(\mathrm{~S}_{0}(s) \vee \mathrm{S}_{1}(s)\right) \Rightarrow \operatorname{SBcAes}(s) \Rightarrow \operatorname{SPE}(s) .
$$

We claim that $S A c B e s \vee S B c A$ s fully characterizes SPE of 0,1-games, in other words.

Conjecture 1. $\forall s,\left(\mathrm{~S}_{0}(s) \vee \mathrm{S}_{1}(s)\right) \Rightarrow(\operatorname{SAcBes}(s) \vee \operatorname{SBcAes} \Leftrightarrow \operatorname{SPE}(s))$.

\section{$5 \quad$ Nash equilibria}

Before talking about escalation, let us see the connection between subgame perfect equilibrium and Nash equilibrium in a sequential game. In [17, the definition of a Nash equilibrium is as follows: A Nash equilibrium is a "pattern [s] of behavior 
with the property that if every player knows every other player's behavior she has not reason to change her own behavior" in other words, "a Nash equilibrium [is] a strategy profile from which no player wishes to deviate, given the other player's strategies." . The concept of deviation of agent $p$ is expressed by a binary relation we call convertibility 4 and we write $\vdash p \dashv$. It is defined inductively as follows:

$$
\begin{gathered}
\frac{s \sim_{s} s^{\prime}}{s \vdash p \dashv s^{\prime}} \\
\frac{s_{1} \vdash p \dashv s_{1}^{\prime} \quad s_{2} \vdash p \dashv s_{2}^{\prime}}{\left\langle\left\langle p, c, s_{1}, s_{2}\right\rangle\right\rangle \vdash p \dashv\left\langle\left\langle p, c^{\prime}, s_{1}^{\prime}, s_{2}^{\prime}\right\rangle\right\rangle} \\
\frac{s_{1} \vdash p \dashv s_{1}^{\prime} \quad s_{2} \vdash p \dashv s_{2}^{\prime}}{\left\langle\left\langle p^{\prime}, c, s_{1}, s_{2}\right\rangle \vdash \vdash p \dashv\left\langle\left\langle p^{\prime}, c, s_{1}^{\prime}, s_{2}^{\prime}\right\rangle\right\rangle\right.}
\end{gathered}
$$

We define the predicate Nash as follows:

$$
\operatorname{Nash}(s) \Leftrightarrow \forall p, \forall s^{\prime}, s \vdash p \dashv s^{\prime} \Rightarrow \widehat{s}(p) \geq \widehat{s^{\prime}}\left(p^{\prime}\right) .
$$

The concept of Nash equilibrium is more general than that of subgame perfect equilibrium and we have the following result:

Proposition 8. $\operatorname{SPE}(s) \Rightarrow \operatorname{Nash}(s)$.

The result has been proven in COQ and we refer to the script (see[15]):

http://perso.ens-lyon.fr/pierre.lescanne/COQ/EscRatAI/

http://perso.ens-lyon.fr/pierre.lescanne/COQ/EscRatAI/SCRIPTS/

Notice that we defined the convertibility inductively, but a coinductive definition is possible. But this would give a more restrictive definition of Nash equilibrium.

\section{Escalation}

Escalation in a game with a set $\mathrm{P}$ of agents occurs when there is a tuple of strategies $\left(s t_{p}\right)_{p \in \mathrm{P}}$ such that its sum is not convergent, in other words, $\neg\left(\bigoplus_{p \in \mathrm{P}} s t_{p}\right) \downarrow$.

Said differently, it is possible that the agents have all a private strategy which combined with those of the others makes a strategy profile which is not convergent, which means that the strategy profile goes to infinity when following the choices. Notice the two uses of a strategy profile: first, as a subgame perfect equilibrium, second as a combination of the strategies of the agents.

Consider the strategy:

$$
\begin{aligned}
& s t_{\mathrm{A}, \infty}=\llbracket r, \llbracket f_{0,1} \rrbracket, s t_{\mathrm{A}, \infty}^{\prime} \rrbracket \\
& s t_{\mathrm{A}, \infty}^{\prime}=\llbracket \mathrm{B}, \llbracket f_{1,0} \rrbracket, s t_{\mathrm{A}, \infty} \rrbracket
\end{aligned}
$$

\footnotetext{
${ }^{4}$ This should be called perhaps feasibility following [18] and 13 .
} 
and its twin

$$
\begin{aligned}
& s t_{\mathrm{B}, \infty}=\llbracket \mathrm{A}, \llbracket f_{0,1} \rrbracket, s t_{\mathrm{B}, \infty}^{\prime} \rrbracket \\
& s t_{\mathrm{B}, \infty}^{\prime}=\llbracket r, \llbracket f_{1,0} \rrbracket, s t_{\mathrm{B}, \infty} \rrbracket .
\end{aligned}
$$

Moreover, consider the strategy profile:

$$
\begin{aligned}
& s_{\mathrm{A}, \infty}=\left\langle\left\langle\mathrm{A}, r,\left\langle\left\langle f_{0,1}\right\rangle\right\rangle, s_{\mathrm{B}, \infty}\right\rangle\right\rangle \\
& s_{\mathrm{B}, \infty}=\left\langle\left\langle\mathrm{B}, r,\left\langle\left\langle f_{1,0}\right\rangle\right\rangle, s_{\mathrm{A}, \infty}\right\rangle\right\rangle .
\end{aligned}
$$

\section{Proposition 9.}

$$
\begin{aligned}
& -s t_{A, \infty} \boldsymbol{\lambda}^{A} \text {, } \\
& \text { - st } t_{B, \infty} \nabla^{B} \text {, } \\
& \text { - } \operatorname{st} 2 \mathrm{~g}\left(s t_{A, \infty}, A\right)=\operatorname{st} 2 \mathrm{~g}\left(s_{B, \infty}, B\right)=G_{0,1} \text {, } \\
& \text { - } \operatorname{game}\left(s_{A, \infty}\right)=G_{0,1} \text {, } \\
& \text { - } s t_{A, \infty} \oplus s t_{B, \infty}=s_{A, \infty} \text {, } \\
& -\neg s_{A, \infty} \downarrow \text {. }
\end{aligned}
$$

Proof. By coinduction.

$s t_{\mathrm{A}, \infty}$ and $s t_{\mathrm{B}, \infty}$ are both rational since they are built using choices, namely $r$, dictated by subgame perfect equilibria 5 which start with $r$. Another feature of this example is that no agent has a clue for what strategy the other agent is using. Indeed after $k$ steps, $A$ does not know if $B$ has used a strategy derived of equilibria in SAcBes or in SBcAes. In other words, A does know if B will stop eventually or not and vice versa. The agents can draw no conclusion of what they observe. If each agent does not believe in the threat of the other she is naturally led to escalation.

\section{Acknowledgements}

The author thanks Samson Abramsky, Franck Delaplace, Stephane Leroux, Matthieu Perrinel, René Vestergaard, Viktor Winschel for their help, encouragements and discussions during this research.

\section{Conclusion}

In this paper, we have shown how to use coinduction in a field, namely economics, where it has not been used yet, or perhaps in a really hidden form, which has to be unearthed. We foresee a future for this tool and a possible way for a refoundation of economics.

\footnotetext{
${ }^{5}$ Our choice of rationality is this of a subgame perfect equilibrium, as it generalizes backward induction, which is usually accepted as the criterion of rationality for finite game.
} 


\section{References}

1. Samson Abramsky and Viktor Winschel. Coalgebraic analysis of subgame-perfect equilibria in infinite games without discounting. arXiv, 2012.

2. Robert J. Aumann. Backward induction and common knowledge of rationality. Games and Economic Behavior, 8:6-19, 1995.

3. Jean-Philippe. Bouchaud. Economics needs a scientific revolution. Nature, 455:1181, oct 2008 .

4. Jean-Philippe Bouchaud and Marc Potters. Theory of Financial Risk and Derivate Pricing. Cambridge University Press, 2003.

5. Venanzio Capretta. Common knowledge as a coinductive modality. In Erik Barendsen, Herman Geuvers, Venanzio Capretta, and Milad Niqui, editors, Reflections on Type Theory, Lambda Calculus, and the Mind, pages 51-61. ICIS, Faculty of Science, Radbout University Nijmegen, 2007. Essays Dedicated to Henk Barendregt on the Occasion of his 60th Birthday.

6. David Colander. The future of economics: the appropriately educated in pursuit of the knowable. Cambridge Journal of Economics, 29:927-941, 2005.

7. Andrew M. Colman. Game theory and its applications in the social and biological sciences. London New York : Routledge, 1999. Second edition.

8. Herbert Gintis. Game Theory Evolving: A Problem-Centered Introduction to Modeling Strategic Interaction. Princeton University Press, 2000.

9. Harold W. Kuhn. Extensive games and the problem of information. Contributions to the Theory of Games II, 1953. Reprinted in [10].

10. Harold W. Kuhn, editor. Classics in Game Theory. Princeton Uni. Press, 1997.

11. Wolfgang Leininger. Escalation and coooperation in conflict situations. J. of Conflict Resolution, 33:231-254, 1989.

12. P. Lescanne and M. Perrinel. From coinduction to the rationality of escalation. http://perso.ens-lyon.fr/pierre.lescanne/PUBLICATIONS/escalation_rational.pdf sep 2009.

13. Pierre Lescanne. Feasibility/desirability games for normal form games, choice models and evolutionary games. CoRR, abs/0907.5469, 2009.

14. Pierre Lescanne. Rationality and escalation in infinite extensive games. CoRR, abs/1112.1185, 2011.

15. Pierre Lescanne and Matthieu Perrinel. "Backward" coinduction, Nash equilibrium and the rationality of escalation. Acta Inf., 49(3):117-137, 2012.

16. Barry O'Neill. International escalation and the dollar auction. J. of Conflict Resolution, 30(33-50), 1986.

17. Martin J. Osborne. An Introduction to Game Theory. Oxford, 2004.

18. Ariel Rubinstein. Lecture Notes in Microecomic Theory. Princeton University Press, 2006.

19. R. Selten. Spieltheoretische Behandlung eines Oligopolmodells mit Nachfrageträgheit. Zeitschrift für gesamte Staatswissenschaft, 121, 1965.

20. Martin Shubik. The dollar auction game: A paradox in noncooperative behavior and escalation. Journal of Conflict Resolution, 15(1):109-111, 1971.

21. Alfred Tarski. A lattice-theoretical fixpoint theorem and its applications. Pacific Journal of Mathematics, 5(2):285309, 1955.

22. Michael Ummels. The complexity of Nash equilibria in infinite multiplayer games. In Roberto M. Amadio, editor, FoSSaCS, volume 4962 of Lecture Notes in Computer Science, pages 20-34. Springer, 2008. 
23. René Vestergaard. A constructive approach to sequential Nash equilibria. Inf. Process. Lett., 97:46-51, 2006.

24. Karl Weierstrass. Über continuirliche Funktionen eines reellen Arguments, die für keinen Werth des letzteren einen bestimmten Differentialquotienten besitzen. in Karl Weiertrass Mathematische Werke, Abhandlungen II Gelesen in der Königl. Akademie der Wisseschaften, am 18 Juli 1872, pages 71-74, 1872.

25. Viktor Winschel. Private communication, 2012.

\section{A Finite 0,1 games and the "cut and extrapolate" method}

We spoke about the "cut and extrapolate" method, applied in particular to the dollar auction. Let us see how it would work on the 0,1-game. Finite games, finite strategy profiles and payoff functions of finite strategy profiles are the inductive equivalent of infinite games, infinite strategy profiles and infinite payoff functions which we presented. Notice that payoff functions of finite strategy profiles are always defined. Despite we do not speak of the same types of objects, we use the same notations, but this does not lead to confusion. Consider two finite families of finite games, that could be seen as approximations of the 0,1 -game:

$$
F_{0,1}=\left\langle\mathrm{A},\left\langle f_{0,1}\right\rangle,\left\langle\mathrm{B},\left\langle f_{1,0}\right\rangle, F_{0,1}\right\rangle\right\rangle \cup\left\{\left\langle f_{0,1}\right\rangle\right\} \quad \begin{aligned}
& K_{0,1}=\left\langle\mathrm{A},\left\langle f_{0,1}\right\rangle, K_{0,1}^{\prime}\right\rangle \\
& K_{0,1}^{\prime}=\left\langle\mathrm{B},\left\langle f_{1,0}\right\rangle, K_{0,1}\right\rangle \cup\left\{\left\langle f_{1,0}\right\rangle\right\}
\end{aligned}
$$

In $F_{0,1}$ we cut after $\mathrm{B}$ and replace the tail by $\left\langle f_{0,1}\right\rangle$. In $K_{0,1}$ we cut after $\mathrm{A}$ and replace the tail by $\left\langle f_{1,0}\right\rangle$. Recall 23 , the predicate $\mathrm{BI}$, which is the finite version of PE.

$$
\begin{aligned}
\mathrm{BI}(\langle f\rangle) & \\
\mathrm{BI}\left(\left\langle p, c, s_{d}, s_{r}\right\rangle\right)= & \mathrm{BI}\left(s_{l}\right) \wedge \mathrm{BI}\left(s_{r}\right) \wedge \\
& \left\langle\left\langle p, d, s_{d}, s_{r}\right\rangle\right\rangle \widehat{s_{d}}(p) \geq \widehat{s_{r}}(p) \wedge \\
& \left\langle\left\langle p, r, s_{d}, s_{r}\right\rangle\right\rangle \Rightarrow \widehat{s_{r}}(p) \geq \widehat{s_{d}}(p)
\end{aligned}
$$

We consider the two families of strategy profiles:

$$
\begin{aligned}
& \mathrm{SF}_{0,1}(s) \Leftrightarrow(i) \Rightarrow\left(s=\left\langle\left\langle\mathrm{A}, d \vee r,\left\langle\left\langle f_{0,1}\right\rangle\right\rangle,\left\langle\left\langle\mathrm{B}, r,\left\langle\left\langle f_{1,0}\right\rangle\right\rangle, s^{\prime}\right\rangle\right\rangle\right\rangle\right\rangle \wedge \mathrm{SF}_{0,1}\left(s^{\prime}\right) \quad \vee\right. \\
& s=\left\langle\left\langle f_{0,1}\right\rangle\right\rangle \\
& \left.\mathrm{SK}_{0,1}(s) \Leftrightarrow-i\right) \Rightarrow s=\left\langle\left\langle\mathrm{A}, r,\left\langle\left\langle f_{0,1}\right\rangle\right\rangle, s^{\prime}\right\rangle\right\rangle \wedge \mathrm{SK}_{0,1}^{\prime}\left(s^{\prime}\right) \\
& \mathrm{SK}_{0,1}^{\prime}(s) \Leftrightarrow(i) \Rightarrow\left(s=\left\langle\left\langle\mathrm{B}, d,\left\langle\left\langle f_{1,0}\right\rangle\right\rangle, s^{\prime}\right\rangle\right\rangle \vee s=\left\langle\left\langle\mathrm{B}, r,\left\langle\left\langle f_{1,0}\right\rangle\right\rangle, s^{\prime}\right\rangle\right\rangle\right) \wedge \mathrm{SK}_{0,1}\left(s^{\prime}\right) \quad \vee \\
& s=\left\langle\left\langle f_{1,0}\right\rangle\right\rangle
\end{aligned}
$$

In $\mathrm{SF}_{0,1}$, B continues and $\mathrm{A}$ does whatever she likes and in $\mathrm{SK}_{0,1}$, A continues and $B$ does whatever she likes. The following proposition characterizes the backward induction equilibria for games in $F_{0,1}$ and $K_{0,1}$ respectively and is easily proved by induction: 


\section{Proposition 10.}

- game $(s) \in F_{0,1} \wedge \mathrm{SF}_{0,1}(s) \Leftrightarrow \mathrm{BI}(s)$,

- game $(s) \in K_{0,1} \wedge \mathrm{SK}_{0,1}(s) \Leftrightarrow \mathrm{BI}(s)$.

This shows that cutting at an even or an odd position does not give the same strategy profile by extrapolation. Consequently the "cut and extrapolate" method does not anticipate all the subgame perfect equilibria. Let us add that when cutting we decide which leaf to insert, namely $\left\langle f_{0,1}\right\rangle$ or $\left\langle f_{1,0}\right\rangle$, but we could do another way around obtaining different results.

0,1 game and limited payroll. To avoid escalation in the dollar auctions, some require a limited payroll, i.e., a bound on the amount of money handled by the agents, but this is inconsistent with the fact that the game is infinite. Said otherwise, to avoid escalation, they forbid escalation. One can notice that, in the 0,1-game, a limited payroll would not prevent escalation, since the payoffs are anyway limited by 1 . 